\title{
Enhancing impedance imaging through multi-modal tomography
}

\author{
Doğa Gürsoy*, Member, IEEE, Yasin Mamatjan ${ }^{\dagger}$, Andy Adler ${ }^{\dagger}$ and Hermann Scharfetter*
}

\begin{abstract}
Several noninvasive modalities including electrical impedance tomography (EIT), magnetic induction tomography (MIT) and induced-current EIT (ICEIT) have been developed for imaging the electrical conductivity distribution within a human body. Although these modalities differ in how the excitation and detection circuitry (electrodes or coils) are implemented, they share a number of common principles not only within the image reconstruction approaches but also with respect to the basic principle of generating a current density distribution inside a body and recording the resultant electric fields. In this paper, we are interested in comparing differences between these modalities and in theoretically understanding the compromises involved, despite the increased hardware cost and complexity that such a multi-modal system brings along. To systematically assess the merits of combining data, we performed 3-D simulations for each modality and for the multi-modal system by combining all available data. The normalized sensitivity matrices were computed for each modality based on the finite element method and singular value decomposition was performed on the resultant matrices. We used both global and regional quality measures to evaluate and compare different modalities. This study has shown that the condition number of the sensitivity matrix obtained from the multi-modal tomography with 16-electrode and 16coil is much lower than the condition number produced in the conventional 16-channel EIT and MIT systems, and thus, produced promising results in terms of image stability. An improvement of about $20 \%$ in image resolution can be achieved considering feasible signal to noise ratio (SNR) levels.
\end{abstract}

Index Terms-Electrical impedance tomography, inducedcurrent EIT, magnetic induction tomography, multi-modal tomography, impedance imaging.

\section{INTRODUCTION}

Several noninvasive modalities have been developed for imaging the electrical conductivity distribution inside a human body. Figure 1 demonstrates the basic principles of three modalities: electrical impedance tomography (EIT), inducedcurrent EIT (ICEIT) and magnetic induction tomography (MIT). The common principles of those modalities are based on generating a current density distribution inside the body and recording the resultant electromagnetic fields by means of sensors positioned around the body. In EIT, the currents are usually applied between pairs of adjacent surface electrodes and the data are collected by measuring the resulting surface potentials [1]. The measured data are then used to reconstruct the cross-sectional images of conductivity (or resistivity) distribution within the body. Several notable features of the

*D. Gürsoy and Hermann Scharfetter are with the Institute of Medical Engineering, Graz University of Technology, Graz, Austria. (e-mail: guersoy@tugraz.at) Asteriks indicates corresponding author.

Y. Mamatjan and A. Adler are with the Department of Systems and Computer Engineering, Carleton University, Ottawa, Canada. device such as high-speed data acquisition, low-cost hardware, portability and noninvasive measurement characteristics make the proposed modality attractive, particularly for monitoring of physiological processes [2]. However, poor skin-electrode contact [3] and electrode position uncertainties [4] yet present a challenge and hinder the practical usage. To evade such contact related issues, ICEIT was proposed as an alternative method for conductivity imaging [5]. ICEIT uses coils to induce currents, and electrodes to record the surface potentials, partly like in EIT. In this way, a higher energy can be applied into the body for the same average current density as in EIT so a higher signal to noise ratio can be achieved [6]. Besides, the number of independent measurements can easily be increased by shifting the coils [7]. However, ICEIT still suffers from problems associated with the electrodes. MIT, on the other hand, offers a completely contactless data acquisition by using coils instead of electrodes for both excitation and detection [8]. Thus, the problems related with the skin electrode contact can readily be eliminated. However, the low dynamic range of measurements [9], image degradation due to the body motions [10] and due to the uncertainties in the coil assembly [11] remain as serious challenges that must be overcome before clinical applications. As it is seen that these techniques differ by how the excitation and detection are implemented, yet the same principle underlies all.

All techniques suffer from the low spatial resolution which is approximately on the order of $10 \%$ to $20 \%$ of the medium diameter near surface regions and far worse at central regions [12]. This is due to the fact that the current density introduced either by coils or by electrodes is typically diffusive. Although increasing the number of measurements by using more coils or electrodes theoretically provides more information on model parameters, which in turn results in an increase in spatial resolution, the practically achievable maximum number of independent measurements is heavily restricted by the measurement noise [13]. Previously, a methodology was proposed to increase the number of independent measurements which appropriately allocates the sources and sensors so that the overall orthogonality between the data is maximized [14]. A new possibility, which we investigated in this paper, is to combine the data acquired from different imaging techniques to increase the number of independent measurements. Although such a multimodal system certainly brings about all the hardware related issues of each modality, we are more interested in determining how much enhancement can be expected from such a multimodal system and to theoretically understand and identify the compromises involved in different modalities. Therefore, we investigated the merits of augmenting data and the possibility 


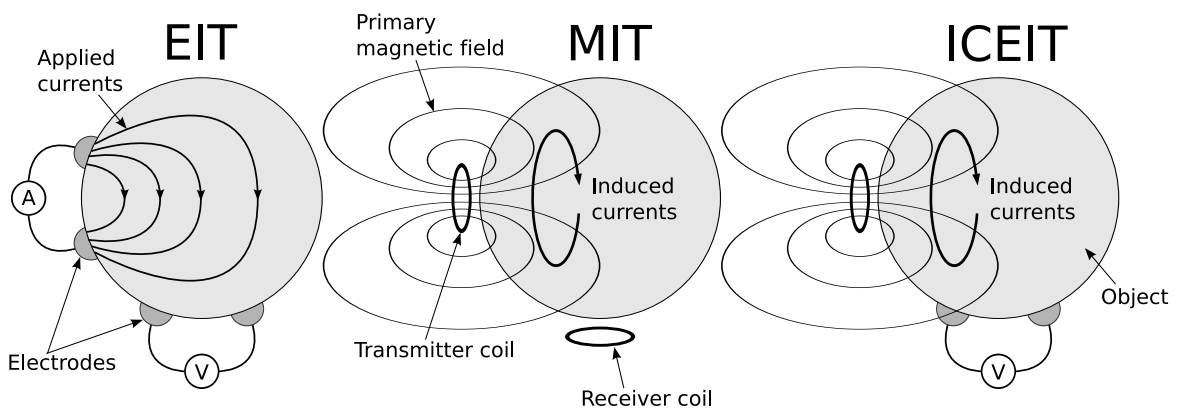

Fig. 1. A comparative demonstration of the basic principles of different impedance imaging modalities: EIT, MIT and ICEIT.

of improving the image quality or ill-conditioning of the problem by fusing the electric and magnetic measurements. To this end, we performed a simulation study considering a homogenous cylindrical phantom as a conducting body. The normalized sensitivity matrices were computed for each technique based on the finite element method and singular value decomposition (SVD) was performed on the resultant matrices. We used systematic approaches to evaluate and compare different modalities. Firstly, based on the singular value spectrum the stability of the truncated-SVD (tSVD) reconstructions were analyzed. Secondly, a radial resolution analysis for the central slice was performed for the underlying techniques. For this, a set of quality measures, which was proposed to quantify cross-sectional EIT images [15], was adopted to evaluate the key features of the point spread functions (PSF). Finally, we displayed the PSFs for some particular locations inside the phantom. This provides an extensive in-depth look into the imaging characteristics of these modalities and helps a better understanding of the compromises and limitations involved.

\section{Methodology FOR MOdeling}

\section{A. Overview of design and considerations}

We systematically investigated the existing coil and electrode based systems used for imaging such as EIT, MIT and ICEIT as illustrated in Figure 1. As described in the introduction, each modality encountered various different challenges related to the experimental design, which limits the achievable spatial resolution. Hereafter, we refer to the modality which makes use of the measurements acquired from the three existing modalities as the multi-modal tomography (MMT). Figure 1 illustrates three possible ways of data acquisition that the coil and electrode based systems can provide to be used for imaging. We focused on the commonly used 16-channel systems with 16 sources and 16 sensors with the following considerations:

Consideration 1: Generally, the complete dataset is obtained by collecting the sensor responses corresponding to each source. Such a data acquisition results in a number of $16 \times 16=256$ available measurements to be used for imaging. However, the reciprocity theorem states that only half of the 256 measurements taken are linearly independent and the remaining are redundant unless they are used for noise averaging to increase the SNR. Note that, we assumed that an active source can also be used as a detector for convenience of performance comparison, although this introduces several difficulties in practice and it is often not desired.

Consideration 2: The sources and detectors of most conventional EIT and MIT systems are assembled on a single ring which encircles the imaging target on a transverse plane. This configuration is possibly the heritage of 2-D EIT imaging and is still the configuration of choice by many groups. However, such configuration brings forth several difficulties, particularly for 3-D imaging, with the most important being the mirroring artifacts. It was reported that due to the complete symmetry of such configurations, the off-plane perturbations are reconstructed on both sides of the central plane [16]. Therefore, in this paper, we placed sensors on two separate rings to prevent the mirroring artifacts. We believe that optimal placement of sources and detectors is crucial for particularly 3-D imaging and there is still need for novel approaches to find configurations that provide better datasets.

Consideration 3: In EIT, an electrode pair is used to inject currents and another electrode pair is used for measurements. Although adjacent current injection and corresponding measurement patterns have been widely used in many EIT systems, other stimulation and measurement patterns (e.g., quasiopposite stimulation patterns) were showed to have better distinguishability than the extensively used adjacent patterns [17]. In this paper, we considered both the adjacent and quasiopposite stimulation patterns to observe the effect on the PSFs. Figure 2 illustrates the corresponding patterns used in this paper.

16 circular electrodes of $1 \mathrm{~cm}$ radii are uniformly distributed on two rings at the surface of a cylindrical phantom with a radius of $10 \mathrm{~cm}$ and a height of $25 \mathrm{~cm}$. In a similar manner, 16 coils of $2.5 \mathrm{~cm}$ radii are radially positioned $2 \mathrm{~cm}$ away from the phantom surface located above the electrodes. We preferred such a two-plane arrangement in order to avoid mirror artifacts which usually appear in the case of radially symmetric measurement assemblies. The corresponding source/detector configurations are depicted in figure 2. After both the coils and the electrodes are available for measurements, one can combine all possible data that these three systems can offer to triple the amount of available measurements. In principle, such a dataset contains all the information about model parameters that EIT, MIT and ICEIT can possibly have and determine the upper limit for the imaging performance. Note that MMT provides a total number of $16 \times 16 \times 3=768$ independent measurements. 


\section{Multi-modal Tomography}

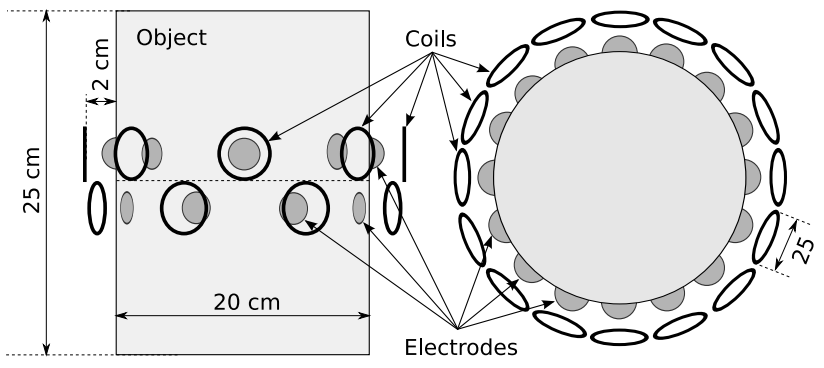

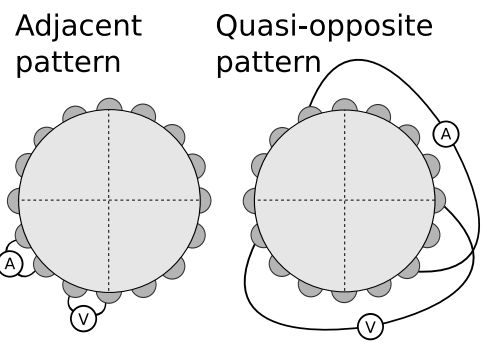

Fig. 2. On the left: the cylindrical phantom and the positioning of measurement configuration. On the right: the measurement and stimulation patterns used for the simulations.

\section{B. Sensitivity formulation}

The image reconstruction problems of EIT, MIT and ICEIT typically consist of partitioning the body into small voxels and calculating the sensitivity of measurements to conductivity changes in each voxel. This requires numerical schemes that can handle arbitrary shaped geometries and conductivity distributions within the body. Finite element discretization [18], [19] is the standard method of choice, but other methods like finite differences [20] and boundary elements [21], [22] have also been studied in detail. A brief review of the formulation is given here to provide a background for the quantitative measures applied to evaluate the quality of reconstructions (refer to [23] for details).

Assume that $\Omega$ represents the conducting medium with the boundary $\partial \Omega$. According to the reciprocity theorem [24], the following expression approximates the change of measurements, i.e., voltage data $\Delta v$, due to a conductivity perturbation $\Delta \sigma$, around a particular distribution $\sigma$ in $\Omega$ :

$$
\Delta v \approx-\frac{1}{I} \int_{\Omega} \Delta \sigma \mathbf{E}_{1}(\sigma) \cdot \mathbf{E}_{2}(\sigma) d V
$$

where $\mathbf{E}_{1}($.$) and \mathbf{E}_{2}($.$) denote the direct and adjoint electric$ fields, respectively. $I$ is the reciprocal current and is usually assumed to be unity. $\sigma$ can be thought of the reference state and generally assumed to be uniform, however, nonuniform distributions can also be used. In this paper, we assumed a phantom with uniform conductivity of $1 \mathrm{Sm}^{-1}$.

In essence, impedance imaging techniques differ by how the electric fields (i.e., $\mathbf{E}_{1}$ and $\mathbf{E}_{2}$ in equation 1) are generated. For instance, for the case of EIT, they are created by applying time-varying currents via pairs of electrodes attached to $\partial \Omega$. Similarly, MIT and ICEIT also use time-varying currents, however through the coils, to induce an electric field in $\Omega$. The formulation and computational details about the electric fields for different techniques can be found in earlier studies [6], [23].

\section{Discretization of the conductivity}

The approximation given by equation 1 must be discretized for computational needs. Generally, $\Omega$ is partitioned into small voxels and a piecewise constant conductivity distribution, i.e., $\sigma=\left[\sigma_{1}, \cdots, \sigma_{N}\right]^{T} \in \mathbb{R}^{N}$, is assumed within $\Omega$. The corresponding expression can be written as,

$$
\Delta v \approx-\frac{1}{I} \sum_{e=1}^{K}\left(\Delta \sigma_{e} \int_{\Omega_{e}} \mathbf{E}_{1}(\sigma) \cdot \mathbf{E}_{2}(\sigma) d v_{e}\right),
$$

where $K$ is the total number of discrete elements, e.g., the number of voxels having a volume element represented by $\Omega_{e}$. By introducing $\mathbf{S}($.$) as the Jacobian operator, the so-$ called sensitivity matrix, equation 2 can be expressed by a linear matrix equation which relates the perturbations in the conductivity distribution to the perturbations in the voltage data,

$$
\Delta v=\mathbf{S}(\sigma) \Delta \sigma
$$

Note that, hereafter, instead of $\mathbf{S}(\sigma), \mathbf{S}$ will be used to denote the sensitivity matrix for convenience. The entries of $\mathbf{S}$ are obtained by successively evaluating the integrals appearing in equation 2 for each voxel corresponding to independent pairs of sources and detectors. In equation $3, \Delta v$ is a vector and each row of $\mathbf{S}$ corresponds to an independent set of voltage data and each column corresponds to a single voxel in the mesh. Note that this approximation is valid only for bounded conductivity fluctuations around $\sigma$ for which the linear approximation error stays below a defined threshold. The validity of this linearity assumption was previously validated with experimental data by reconstructing single or multiple perturbations with sizes on the order of $10 \%$ of the medium diameter [25].

\section{Normalization of the sensitivity matrix}

The excitation parameters, i.e., the operating frequency and the amplitude of the source currents, show differences depending on whether electrodes or coils are used. For instance, in the case of EIT, the applied currents are typically several milliamps with operating frequencies on the order of several $\mathrm{kHz}$. On the other hand, for the case of MIT, the signal amplitudes can reach values as high as several amperes and the frequency goes up to several MHz. This also affects the measurements and the corresponding SNR levels. Thus, the computed sensitivity matrices must be adjusted for the comparison to be valid. For this, we normalized the sensitivities as follows. By denoting $s_{i, j}$ as the coefficients of 
$\mathbf{S}$, each sensitivity matrix is normalized with the constant $\eta$ :

$$
\eta=\frac{1}{N K}\left(\sum_{i}^{N} \sum_{j}^{K} s_{i, j}^{2}\right)^{1 / 2},
$$

where $N$ and $K$ simply denote the number of measurements and the voxels in the finite element mesh, respectively. The normalized sensitivity matrices will be used for quality evaluations throughout the rest of the paper.

\section{EVALUATION PARAMETERS}

SVD based analysis (e.g., analysis of the decay of singular values) provides global measures about the reconstruction quality and does not provide much about the regional information. Thus, in addition to a SVD analysis, we applied a number of quality measures that evaluates the performances on the radial axis. Besides, we presented the PSF distributions at specific locations to investigate the regional effects. Different regularization levels were selected in order to investigate the practical and theoretical limits. For instance, $60 \mathrm{~dB}$ tSVD regularization was used to evaluate the practical performances, since, $60 \mathrm{~dB}$ is the maximum achievable SNR of the current systems. On the other hand, $120 \mathrm{~dB}$ tSVD regularization was preferred to investigate the theoretical limits of the modalities. Note that even for simulating the theoretical case, we had to apply a small amount of regularization to suppress the computational errors originated from the FE solver.

\section{A. SVD based analysis and reconstruction}

We used SVD which is a conventional tool for the analysis and comparison of such linear inverse problems. It provides not only the reconstructed parameters but also a framework for the quantification of key properties such as the resolution and stability of the reconstructions [16]. By applying SVD to $\mathbf{S}$, we get $\mathbf{U} \Sigma \mathbf{V}^{T}$, where $\mathbf{U}$ and $\mathbf{V}$ are the singular vectors which form a basis for the data and the model space (conductivity distribution in this case), respectively, and $\Sigma$ is a diagonal matrix, the diagonal entries of which are called the singular values. The conductivity parameters can be reconstructed by calculating the generalized inverse $\mathbf{S}^{\dagger}$ which can be expressed in terms of singular components as $\mathbf{V} \Sigma^{\dagger} \mathbf{U}^{T}$, where $\Sigma^{\dagger}$ is the pseudo-inverse of $\mathbf{S}$ with every nonzero entry replaced by its reciprocal. It is apparent that the small singular values yield instability in reconstructions and must be suppressed. Generally, a truncation by using only the $t$ column vectors of $\mathbf{U}$ and $\mathbf{V}$ corresponding to the $t$ largest singular values is preferred to stabilize the inversion when the data are inaccurate. This improves the condition number and in turn mitigates the ill-conditioning of $\mathbf{S}$. The solution can eventually be computed from,

$$
\Delta \sigma=\mathbf{V} \Sigma_{t}^{\dagger} \mathbf{U}^{T} \Delta v
$$

where the subscript $t$ is defined as the truncation index (or regularization parameter) that determines the stability of the inversion by ignoring the $n-t$ number of small singular values in $\Sigma$ and the associated singular vectors in $\mathbf{U}$ and $\mathbf{V}$.
There are several ways to select $t$ (e.g., L-curve, discrepancy principle, generalized cross validation, etc. [26]). In this paper, $t$ was selected based on the definition of SNR as explained follows. Let $\rho_{1}$ be the largest singular value and $\rho_{t}$ the $t^{t h}$ largest one, the proper selection of the truncation level $t$ was established by choosing the maximum $t$ that satisfies the following equality [27],

$$
S N R=20 \log _{10}\left(\frac{\Delta v_{r m s}}{\delta_{r m s}}\right) \geq 20 \log _{10}\left(\frac{\rho_{1}}{\rho_{t}}\right)
$$

where $\Delta v_{r m s}$ and $\delta_{r m s}$ are the root mean square of the perturbed voltage signal and the noise signal, respectively. The left hand side of the inequality is defined as the SNR. If the noise is modeled as an additive white Gaussian noise of zero mean, $\delta_{r m s}$ simply refers to standard deviation of the noise. Note that, in numerical analysis, $\rho_{1} / \rho_{t}$ in equation 6 is commonly referred to as the condition number and is a measure of the ill-conditioning of the problem.

\section{B. Quality measures}

Previous sections clarified how the reconstructions can be obtained from the sensitivity matrix as a means of applying singular value decomposition. This section, however, is about the measures that quantify the output images. The linear inverse theory, permits us to attain various local or regional quality measures with regard to the resolvability of conductivity parameters. For that purpose, a quantification of the resolution matrix mean in terms of point spread functions is the widely utilized method of choice. This allows obtaining the resolution limits of the reconstructions without even having measurements. The resolution matrix can simply be defined as $\mathbf{R}=\mathbf{S}^{\dagger} \mathbf{S} \in \mathbb{R}^{N \times N}$ with $N$ being the number of model parameters. Ideally, all true parameters can be perfectly resolved if $\mathbf{R}$ is an identity matrix; however, in practical applications due to the limited amount of data and nonzero measurement noise, $\mathbf{R}$ is far from an identity matrix. The columns of $\mathbf{R}$ are commonly referred to as the point spread functions (PSF). In terms of SVD components resolution matrix can be calculated from:

$$
\mathbf{R}=\mathbf{V} \Sigma^{\dagger} \Sigma \mathbf{V}^{T} .
$$

Therefore, the resolution is simply $\mathbf{V V}^{T}$ considering only the singular vectors corresponding to the nonzero singular values determined by the truncation index $t$.

Although the complete analysis of the resolution matrix by browsing through each PSF of individual voxels is highly informative, it is non-trivial in practice due to the large number of parameters. Practical measures to quantify the key features of the PSFs are desired for performance evaluation. Therefore, to evaluate the performance of these different modalities, we used a set of evaluation criteria defined in GREIT (Graz consensus Reconstruction algorithm for EIT) [15] which are in order of importance: amplitude response (AR), position error (PE), resolution (RES) and shape deformation (SD).

We characterized these modalities through the quality measures based on the selected PSF as the image of a point conductivity change (i.e., the conductivity change of a single voxel), which is much smaller than the resolution of EIT, MIT 
and ICEIT with 16 channels. We used 2-D cross-sectional images of the PSFs for the evaluation and we refer to the position of the perturbed voxel as the target position. The location of the PSF peak is called the center of gravity (CoG). Hereafter, the PSF is represented by the discrete vector $\hat{\mathbf{x}}$. We considered the quarter maximum amplitude set, i.e., $\hat{\mathbf{x}}_{q} \geq 0.25 \max (\hat{\mathbf{x}})$, as the region of interest (ROI). In the following, we briefly summarize the quality measures and explain how they can be calculated efficiently:

1) Amplitude Response $(A R)$ is defined as the maximum amplitude of the PSF divided by the associated voxel area $V_{T}$. It can be written as:

$$
\mathrm{AR}=\max (\hat{\mathbf{x}}) / V_{T},
$$

$\mathrm{AR}$ is regarded as the most important figure of merit to decide the image quality at GREIT, which depends on the accuracy of each measurement. It is desirable for AR to be uniform over the range of target positions.

2) Position Error $(P E)$ is used to measure the extent of dislocation of the PSF peak from the target position. It is calculated as the discrepancy between $r_{q}$ (the centre of gravity of the ROI) and $r_{t}$ (the original position of the target):

$$
\mathrm{PE}=r_{t}-r_{q}
$$

It is important for PE to be small, because large PE may lead to unreliable interpretation of images.

3) Resolution (RES) is used to measure the smallest visible object within a system that reflects the system resolution. It is calculated based on the ratio of the area of ROI $\left(A_{q}\right)$ to the area of entire cross-section $\left(A_{0}\right)$ and can be expressed as:

$$
\mathrm{RES}=\sqrt{A_{q} / A_{0}}
$$

RES measures radius ratios rather than area ratios.

4) Shape Deformation (SD) quantifies the artifacts that often appear for the target positions placed near the electrodes or tank boundary. Those strange shaped artifacts may lead to imaging artifacts and thus incorrect interpretation of images. SD can be calculated as,

$$
\mathrm{SD}=\sum_{k \notin C}\left[\hat{\mathbf{x}}_{q}\right]_{k} / \sum_{k}\left[\hat{\mathbf{x}}_{q}\right]_{k},
$$

where $C$ is a circle centered at the CoG of $\hat{\mathbf{x}}_{q}$ with an equivalent area of $A_{q}$.

In general, it is desirable for AR, PE, RES and SD to be constant, while PE, RES and SD should be as small as possible for any target position. AR and PE are regarded as the most important figures of merit [15].

\section{Simulation protocol for quality measures}

Considering uniformly distributed electrodes and coils on the surface, the differences in PSFs at a fixed radial distance are marginal. Therefore, we investigated the PSF characteristics on the radial axis. For a radial evaluation, the central transverse slice, i.e. at $\mathrm{z}=0$, was divided into tiny coaxial rings of different radii starting from the origin to the periphery of the slice as shown in figure 3. The thickness and height of the rings were selected as $1.25 \mathrm{~cm}$ and $5 \mathrm{~cm}$, respectively. Recall that the cylindrical phantom has a radius of $10 \mathrm{~cm}$ and a height of $25 \mathrm{~cm}$. The measures for the PSFs corresponding to the perturbation at each voxel were calculated and averaged over the ring that they were associated. A detailed illustration describing the computations is given in figure 3 and the steps taken are briefly summarized below:

1) The sensitivity matrices of EIT, MIT, ICEIT and MMT were computed based on a uniform conductivity distribution within the cylindrical phantom and were normalized based on equation 4.

2) The PSFs located at each voxel of the finite element mesh (i.e., resolution matrices of the modalities) were calculated and the corresponding quality measures were computed for each PSF.

3) The phantom is divided into ring shaped regions of different radii for a radial evaluation and the computed quality measures were averaged over the ring volume that they were associated.

As can be noted from equation 7, the PSFs are strictly dependent on the regularization applied. Thus, we calculated two sets of PSFs considering different levels of truncation of the singular vectors. One is computed for a $120 \mathrm{~dB}$ SNR and the other is for $60 \mathrm{~dB}$ SNR according to equation 6 and, hereafter, these two cases will be referred to as $120 \mathrm{~dB}$ and $60 \mathrm{~dB}$ tSVD regularizations, respectively.

\section{RESUlts}

\section{A. Sensitivity maps}

The sensitivity maps give ideas about the imaging characteristics and suggests how the electrodes and sensors can be positioned effectively. Figure 4 displays the calculated sensitivity distributions of EIT, MIT and ICEIT systems for particular sources and detector pairs. The locations of the source and detector pairs are explicitly shown for all systems. For the case of EIT, two neighboring electrodes are used to inject currents and an oppositely located electrode pair is used to measure surface voltages. Sharp peaks can be noted at the proximity of the electrode locations. However, the sensitivity map of MIT is found to be spatially smoother than that of EIT. For both cases, large positive and negative sensitivities are noted, particularly near-boundary zones. For the case of ICEIT, the sensitivity is found to be zero for the cross-sectional plane, which is expected due to the special symmetry of the arrangement. That's one of the reasons that we did not consider coil and electrode configurations on a single plane. Another reason was to avoid mirroring artifacts that are usually observed for such symmetric configurations.

\section{B. Singular value analysis}

After SVD had been performed on the sensitivity matrices, the truncation levels required for a stable image reconstruction were determined using the logarithmic ratios, i.e., $20 \log _{10}\left(\rho_{1} / \rho_{t}\right)$, between the first and the remaining singular values as compared to the SNR level as shown in figure 5. The ascend of the curves indicate key properties of 


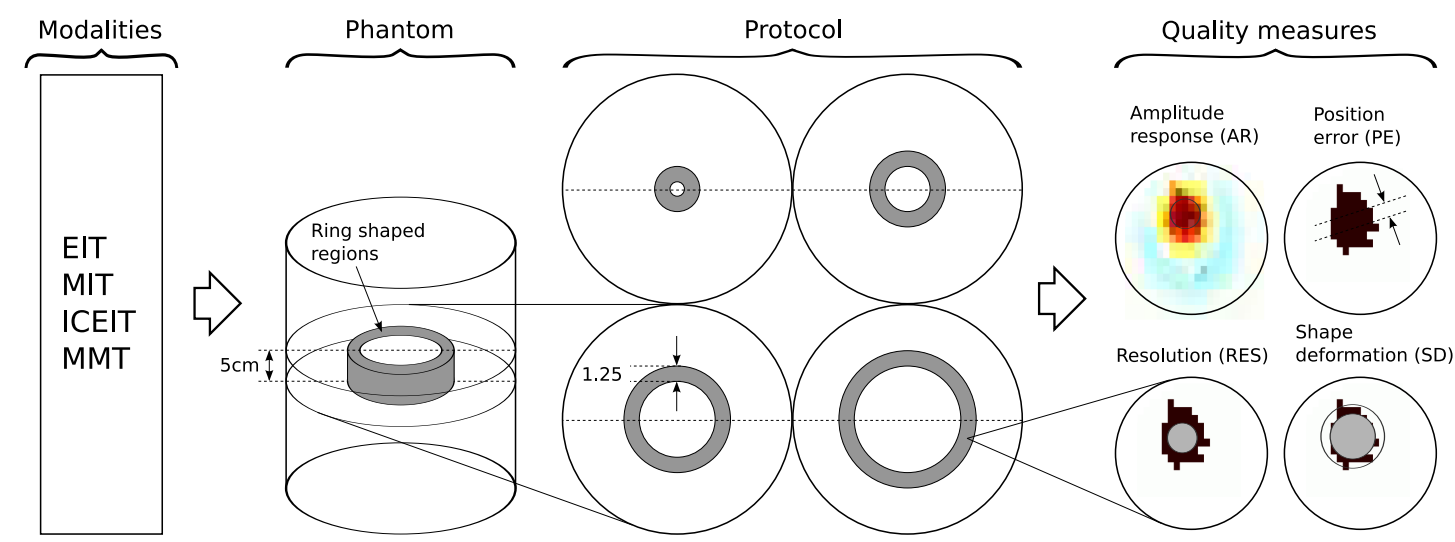

Fig. 3. Demonstration of how the radial analysis of quality measures is performed. The phantom was partitioned into coaxial rings of different radii starting from the origin to the boundary. Each ring has a fixed $1.25 \mathrm{~cm}$ thickness and $5 \mathrm{~cm}$ height. The measures for the perturbation at each voxel within the ring are calculated and averaged over the ring.

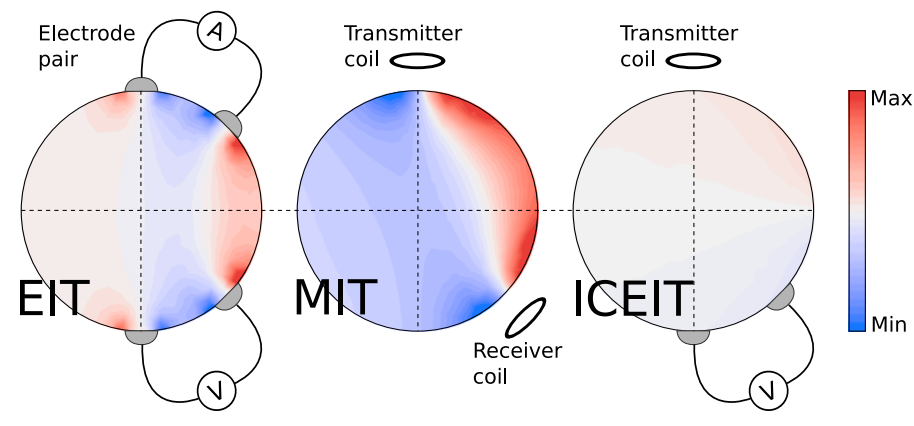

Fig. 4. The sensitivity distributions on the central plane for EIT, MIT and ICEIT. Note that, for the case of ICEIT, the sensitivity is zero for the cross-sectional plane, which is expected due to the special symmetry of the single-plane arrangement (That's why we considered coil and electrode configurations on two separate planes).

the inversion such as imaging stability under noisy data. The curve corresponding to EIT ascends more steeply than others and therefore reflects a faster drop of the singular values. For EIT, only 92 out of a total number of 256 singular values can effectively be used for a stable image reconstruction considering $60 \mathrm{~dB}$ tSVD regularization. On the other hand, the curve of MMT ascends with a smoother slope and the truncation index is significantly larger considering SNR levels below $120 \mathrm{~dB}$. This clearly indicates improved conditioning of the inversion and reconstruction stability. In addition, the continuous and dashed curves in figure 5 represent the adjacent and quasiopposite stimulation and measurement patterns, respectively. The curves for the adjacent pattern were found to ascend more steeply than the quasi-opposite pattern at start and then it ascends more slowly. However, the effect of different patterns on the singular values were found to be marginal as compared with the singular values of different modalities.

\section{Quality distributions}

Figure 6 displays the resulting quality measure plots of the cross-sectional PSFs (in order of importance) along the radial axis for two different regularization applied (i.e., different truncation levels). The plots in the left and right columns represent the cases for $120 \mathrm{~dB}$ and $60 \mathrm{~dB}$ tSVD regularizations

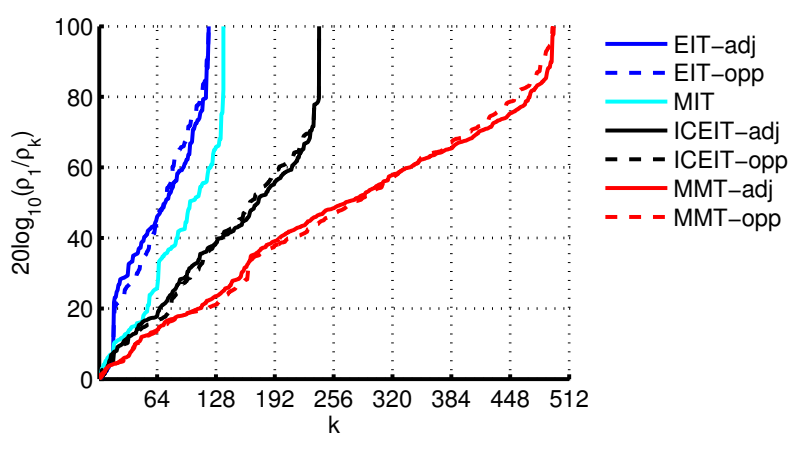

Fig. 5. Y-axis represents the logarithmic ratio between the first and the kth singular value in $\mathrm{dB}$ (i.e., the condition number of the sensitivity matrices in $\mathrm{dB}$ ). $\mathrm{k}$ represents the measurement index. Only the values below $100 \mathrm{~dB}$ are shown. The abbreviations adj and opp represents the adjacent and quasiopposite stimulation and measurement patterns, respectively.

and they represent the imaging performances for the case of low-noise and high-noise, respectively. The corresponding simulation protocol is described in the "simulation protocol for quality measures" section, previously and illustrated in figure 3.

It was found that the AR value of the hybrid method is approximately double the values produced by other methods. It clearly indicates a larger peak of the PSF in the hybrid method for both cases of $120 \mathrm{~dB}$ and $60 \mathrm{~dB}$ tSVD regularizations. The shape of the AR plots showed similar patterns for all modalities, so we calculated the dynamic range which is the ratio between the maximum and minimum AR values. The dynamic ranges for EIT, MIT, ICEIT and MMT are found to be 3.3, 4, 5.8 and 3 for the case of $120 \mathrm{~dB}$ tSVD regularization and $3.5,4,3.4$ and 2.8 for for $60 \mathrm{~dB}$ tSVD regularization, respectively. The lower dynamic range means smoother curve with more constant values and AR also should be constant for any PSF position. The results showed that AR of MMT has the least dynamic range among all.

The PEs of all methods are found to increase with the radial distance. The PE curve of MMT is the most constant and the average PE of MMT over the entire cross-section is about two times smaller than the PEs of other modalities for both $120 \mathrm{~dB}$ 

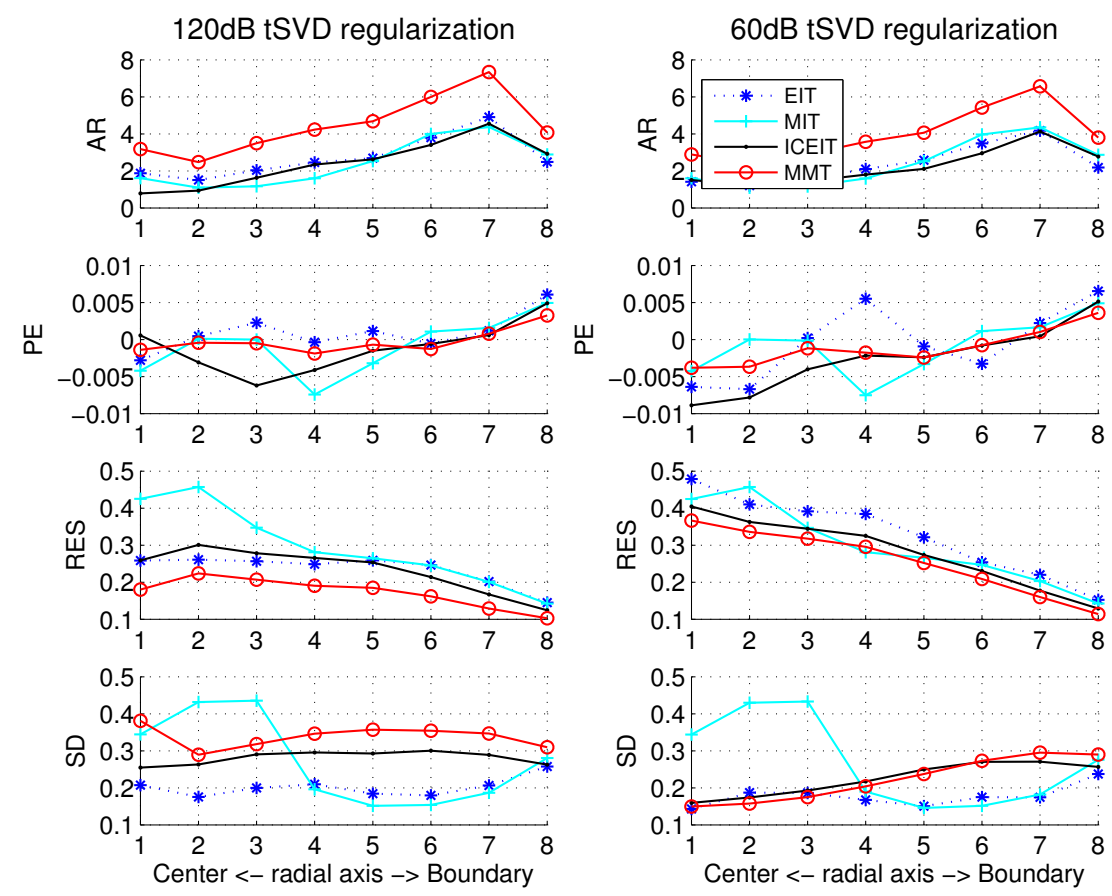

Fig. 6. The plots of the average quality measures along the radial axis. Y-axes refer to the quality measures and $\mathrm{x}$-axes refer to the index of the rings with different radii ( 1 denotes the central ring and 8 denotes the ring close to the boundary). The left and right columns represent the cases for $120 \mathrm{~dB}$ and $60 \mathrm{~dB}$ tSVD regularizations, respectively.

and $60 \mathrm{~dB}$ tSVD regularizations. The effect of regularization on the PE values of MIT is found to be marginal.

The RES plots show that $60 \mathrm{~dB}$ tSVD regularization results in high RES values particularly at the central locations, except for the case of MIT. The RES of MMT is more uniformly distributed from center to the edge and smallest, which are the desired behavior. Considering zero truncation, MMT produces RES values about 0.18 at central regions and 0.10 at regions close to the boundary whereas the best among other modalities produces 0.26 for inner and 0.17 for near-boundary regions. Considering $60 \mathrm{~dB}$ tSVD regularization, MMT shows approximately \%10-20 lower RES values than others.

The SD of MMT is very similar to that of ICEIT, particularly for $60 \mathrm{~dB}$ tSVD regularization. The SD values of MIT near central regions are found to be very high. The SD of EIT is very low compared to others.

\section{Point spread functions}

For a detailed investigation, the PSFs at six specific locations were computed for both $120 \mathrm{~dB}$ and $60 \mathrm{~dB}$ tSVD regularizations in order to analyze the imaging characteristics of the modalities. The corresponding PSFs are depicted in figure 7 and figure 8 . The coronal and transversal images were chosen to cut through the object for particular target locations. For convenience, hereafter, the PSFs corresponding to different locations will be referred by the labels given in bottom-left of the images (i.e, $P 1, \cdots, P 6$ ). For instance, P1-MIT will refer to the PSF of MIT at location $[3.33,0,6.66] \mathrm{cm}$. From the images, the PSFs located close to the z-axis (i.e., P1, P2 and P3) provide more diffused characteristics as opposed to the locations close to the surface (i.e., P4, P5 and P6). The position errors of the PSFs are relatively low for P5 and P6, however, for P4 the localization errors become visible, particularly for EIT and MIT. With increasing distance from the sensor locations, the PSFs show more blurry characteristics. P5 and P6 produced the most accurate and reasonable results with regard to the peak location and shape. Although, not visible from the colormap, the more blurry the distribution the less the peak amplitude of the PSF is. The PSFs computed with all singular values and vectors show better localization and resolution compared to PSFs corresponding to $60 \mathrm{~dB}$ tSVD regularization, particularly at central regions.

\section{DISCUSSIONS AND CONCLUSIONS}

Our primary motivation was to investigate how much additional performance improvements can be expected by combining datasets of different modalities. Therefore, we compared the imaging characteristics of 16-channel conventional EIT, MIT and ICEIT systems, and combined them to investigate how much the imaging quality of the systems can be enhanced. The analysis is mainly based on the evaluation of the PSFs using a set of quality measures.

In general, the cross-sectional reconstructions are imaged rather than visualizing the whole 3-D reconstructions. Therefore, for the calculation of the measures, we calculated the complete 3-D PSF at first, however used the 2-D transverse PSFs at the target voxel locations. It was shown that the condition number of such a hybrid system (MMT) with 16 pairs of electrodes and coils is much lower than the conventional 


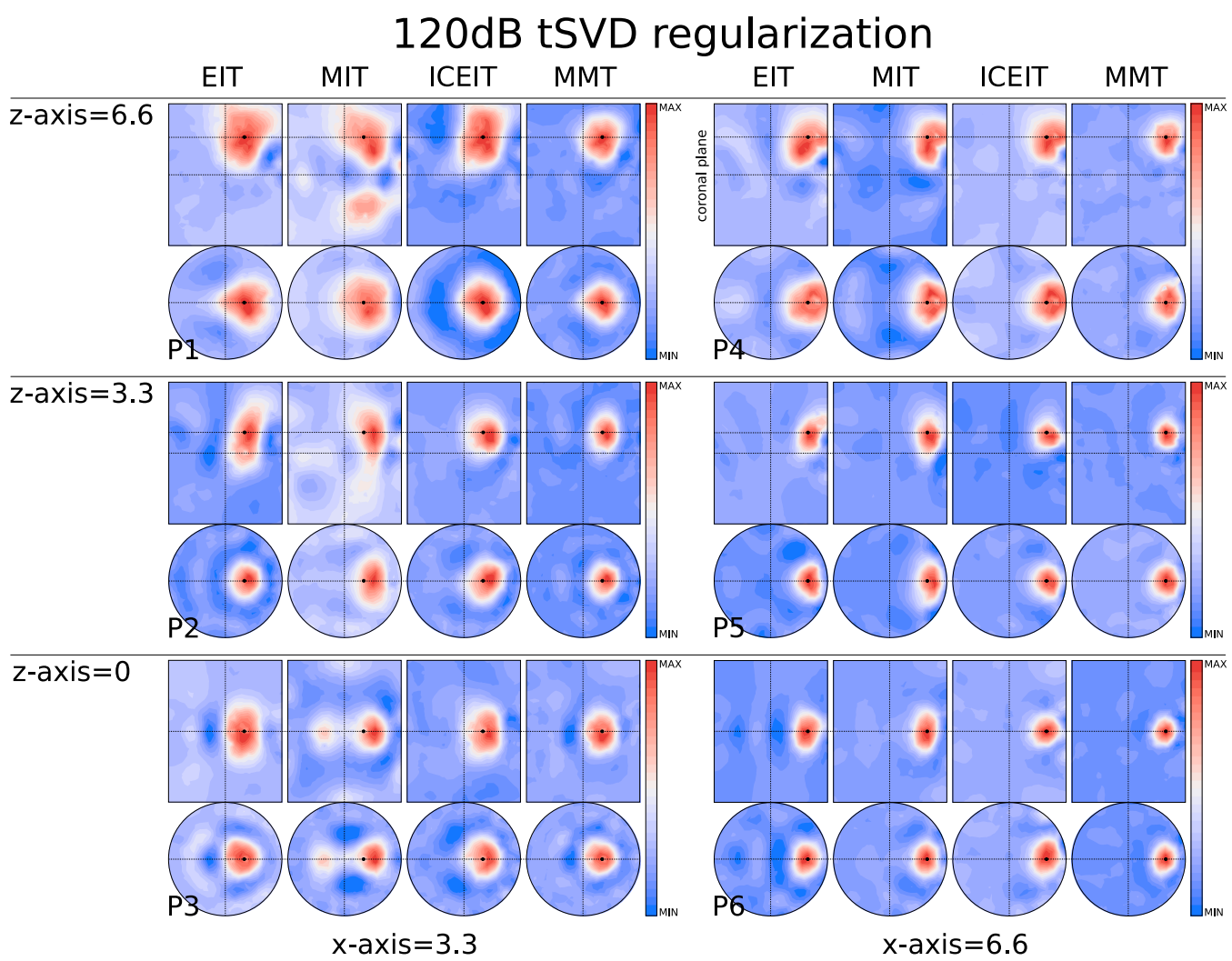

Fig. 7. The PSFs for different measurement techniques considering $120 \mathrm{~dB}$ tSVD regularization. The small dots on the images represent the target positions of the PSFs. MAX and MIN values on the colorbar represent the maximum and minimum values for each image.

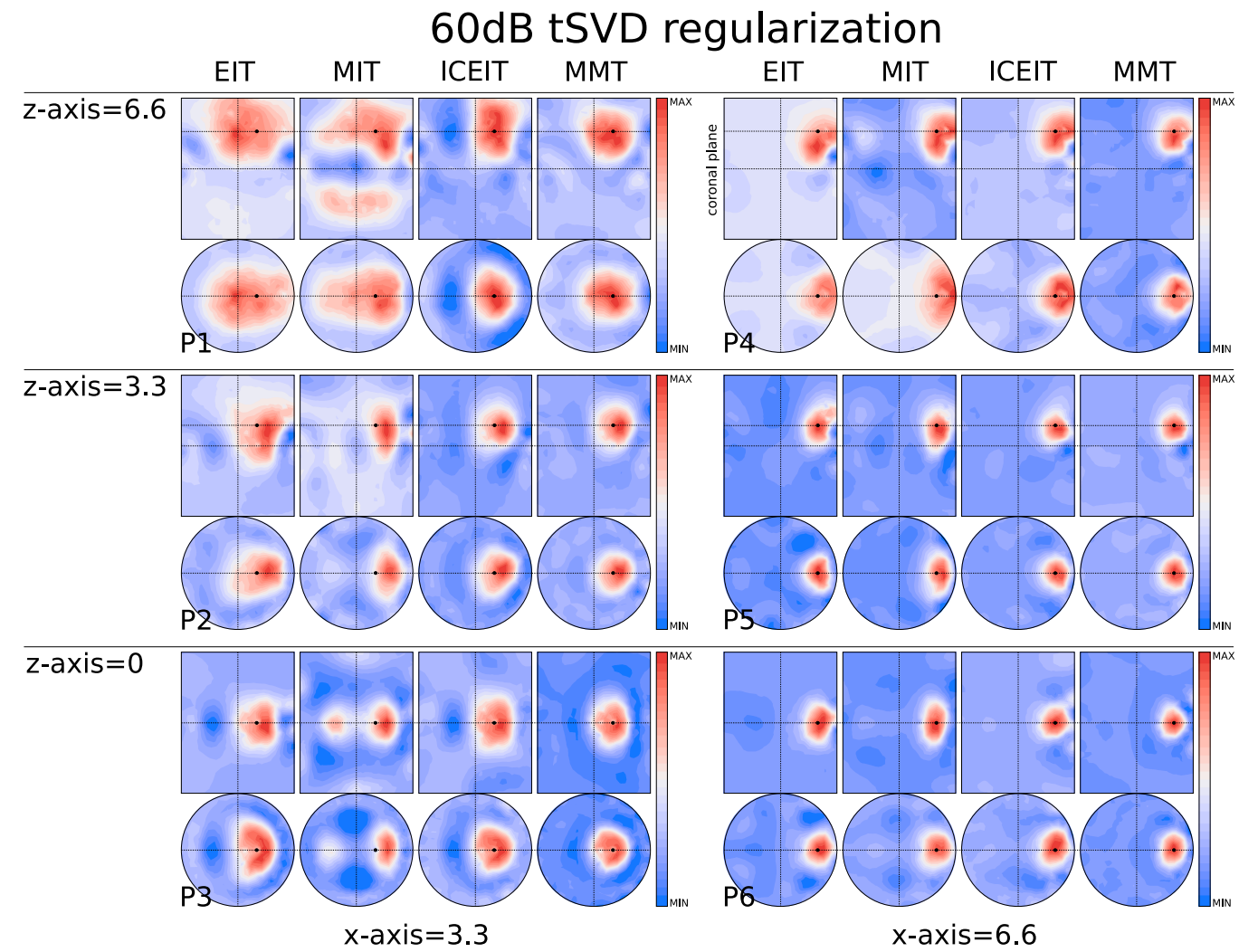

Fig. 8. The PSFs for different measurement techniques considering $60 \mathrm{~dB}$ tSVD regularization. The small dots on the images represent the target positions of the PSFs. MAX and MIN values on the colorbar represent the maximum and minimum values for each image. 
16-channel EIT and MIT systems and thus, shows promising results in terms of image stability.

The quality measures are preferred to quantify the key features of the PSFs to characterize these modalities. The PSFs were calculated considering $120 \mathrm{~dB}$ and $60 \mathrm{~dB}$ tSVD regularizations to investigate the theoretical and practical limits of image qualities, respectively. All modalities showed similar AR plots, where MMT yielding the highest values with more uniformly distributed values. MMT also yields a more constant PE and RES plots with smaller values than those of other modalities for both regularizations applied. This clearly indicates that MMT offers an improved imaging performance and can be an alternative to the existing modalities.

As a final evaluation, the PSFs in several particular locations inside the phantom is calculated to provide an extensive indepth look into the imaging characteristics of these modalities at both the coronal and transversal planes. It was noticed from figures 7 and 8 that the images become more blurry with increasing distance between target and sensors. The $120 \mathrm{~dB}$ tSVD regularized images show better localization and resolution properties than $60 \mathrm{~dB}$ tSVD regularized images, particularly at central regions. The performance of EIT and MIT is found to be poor overall, particularly for central regions, however, MMT and ICEIT showed promising results.

ICEIT was proposed as an alternative method for conductivity imaging [28], [29], yet, with a completely different coil configuration compared to the one we used in this paper. Conventional ICEIT systems use typically several large coils with their axis perpendicular to the imaged cross-section to be used as sources. This arrangement has been used to obtain improved resolution at the central locations as compared with the EIT [30]. However, it is not possible to have a sensitive zone with the single plane configurations as proposed in this paper. On the other hand, the two-plane configurations also favored ICEIT over EIT for reasonable levels of SNR in terms of resolution. Nevertheless, ICEIT outperforms EIT also in the plane analyzed in this paper.

In this paper, we consider normalization based on the $L_{2^{-}}$ norm of the the sensitivity matrix for the comparisons. With this approach the systems can be assessed using only the sensitivity matrices even without having measurements and renders possible to understand the theoretical limits of the imaging performances. Alternatively, another option would be normalizing by the noise inherent in the modality. For instance, introducing data covariance matrices to be used for regularization [13] and make inferences on the resulting images.

We conclude that electrical and magnetic measurements complement each other and can be used to gather additional information about the model parameters. Such a multi-modal dataset results in an improvement of about $20 \%$ in spatial resolution for feasible levels of SNR. Although the proposed multi-modality system provides more opportunities to improve image quality, it may comprise all the hardware drawbacks of electrode and coil based measurement systems. In this regard, body motions should still be tracked during data acquisition, the dynamic range of the receiving circuits must be large enough to record the signals due to the conductivity perturbations and electrode artifacts must be considered.

\section{REFERENCES}

[1] D. C. Barber and B. H. Brown, "Applied potential tomography," Journal of Physics E: Scientific Instruments, vol. 17, no. 9, pp. 723-733, 1984.

[2] T. Muders, H. Luepschen, and C. Putensen, "Impedance tomography as a new monitoring technique," Current Opinion in Critical Care, vol. 16, no. 3, pp. 269-275, 2010

[3] K. G. Boone and D. S. Holder, "Effect of skin impedance on image quality and variability in electrical impedance tomography: a model study," Medical and Biological Engineering and Computing, vol. 34, no. 5, pp. 351-354, 1996.

[4] M. Soleimani, C. Gomez-Laberge, and A. Adler, "Imaging of conductivity changes and electrode movement in EIT," Physiological Measurement, vol. 27, no. 5, pp. 103-113, 2006.

[5] W. R. Purvis, R. C. Tozer, D. K. Anderson, and I. L. Freeston, "Induced current impedance imaging," Science, Measurement and Technology, IEE Proceedings A, vol. 140, no. 2, pp. 135-141, 1993.

[6] S. Zlochiver, M. M. Radai, S. Abboud, M. Rosenfeld, X. Dong, R. Liu, F. You, H. Xiang, and X. Shi, "Induced current electrical impedance tomography system: experimental results and numerical simulations," Physiological Measurement, vol. 25, no. 1, pp. 239-255, 2004.

[7] N. G. Gençer, Y. Z. İder, and S. J. Williamson, "Electrical impedance tomography: induced-current imaging achieved with a multiple coil system," Biomedical Engineering, IEEE Transactions on, vol. 43, no. 2, pp. 139-149, 1996.

[8] H. Griffiths, "Magnetic induction tomography," Measurement Science and Technology, vol. 12, no. 8, pp. 1126-1131, 2001.

[9] H. Griffiths, W. R. Stewart, and W. Gough, "Magnetic induction tomography: A measuring system for biological tissues," Annals of the New York Academy of Sciences, vol. 873, no. 1, pp. 335-345, 1999.

[10] D. Gürsoy and H. Scharfetter, "Reconstruction artefacts in magnetic induction tomography due to patient's movement during data acquisition," Physiological Measurement, vol. 30, no. 6, pp. 165-174, 2009.

[11] D. Gürsoy and H. Scharfetter, "Imaging artifacts in magnetic induction tomography caused by the structural incorrectness of the sensor model," Measurement Science and Technology, vol. 22, no. 1, p. 015502, 2011.

[12] D. S. Holder, Ed., Electrical impedance tomography: Methods, history and applications. Bristol: Institute of Physics Publishing, 1995.

[13] A. Tarantola, Inverse Problem Theory and Model Parameter Estimation. Philadelphia: SIAM, 2005.

[14] D. Gürsoy and H. Scharfetter, "Optimum receiver array design for magnetic induction tomography," Biomedical Engineering, IEEE Transactions on, vol. 56, no. 5, pp. 1435-1441, 2009.

[15] A. Adler, J. H. Arnold, R. Bayford, A. Borsic, B. Brown, P. Dixon, T. J. C. Faes, I. Frerichs, H. Gagnon, Y. Gärber, B. Grychtol, G. Hahn, W. R. B. Lionheart, A. Malik, R. P. Patterson, J. Stocks, A. Tizzard, N. Weiler, and G. K. Wolf, "GREIT: a unified approach to 2d linear EIT reconstruction of lung images," Physiological Measurement, vol. 30, no. 6 , pp. $35-55,2009$.

[16] D. Gürsoy and H. Scharfetter, "The effect of receiver coil orientations on the imaging performance of magnetic induction tomography," Measurement Science and Technology, vol. 20, no. 10, p. 105505 (9pp), 2009.

[17] A. Adler, P. O. Gaggero, and Y. Maimaitijiang, "Adjacent stimulation and measurement patterns considered harmful," Physiological Measurement, 2011.

[18] K. Hollaus, C. Magele, R. Merwa, and H. Scharfetter, "Fast calculation of the sensitivity matrix in magnetic induction tomography by tetrahedral edge finite elements and the reciprocity theorem," Physiological Measurement, vol. 25, no. 1, pp. 159-168, 2004.

[19] M. Soleimani, W. R. B. Lionheart, A. J. Peyton, X. Ma, and S. R. Higson, "A three-dimensional inverse finite-element method applied to experimental eddy-current imaging data," Magnetics, IEEE Transactions on, vol. 42, no. 5, pp. 1560-1567, 2006.

[20] A. Morris, H. Griffiths, and W. Gough, "A numerical model for magnetic induction tomographic measurements in biological tissues," Physiological Measurement, vol. 22, no. 1, pp. 113-119, 2001.

[21] M. H. Pham and A. J. Peyton, "Computation of 3-D sensitivity coefficients in magnetic induction tomography using boundary integral equations and radial basis functions," Magnetics, IEEE Transactions on, vol. 44, no. 10, pp. 2268-2276, 2008.

[22] S. Engleder and O. Steinbach, "Boundary integral formulations for the forward problem in magnetic induction tomography," Mathematical Methods in Applied Sciences, vol. 34, no. 9, pp. 1144-1156, 2011. 
[23] M. Soleimani, "Computational aspects of low frequency electrical and electromagnetic tomography: A review study," International Journal of Numerical Analysis and Modeling, vol. 5, no. 3, pp. 407-440, 2008.

[24] J. R. Mortarelli, "A generalization of the geselowitz relationship useful in impedance plethysmographic field calculations," Biomedical Engineering, IEEE Transactions on, vol. BME-27, no. 11, pp. 665-667, 1980.

[25] R. Merwa, P. Brunner, A. Missner, K. Hollaus, and H. Scharfetter, "Solution of the inverse problem of magnetic induction tomography (MIT) with multiple objects: analysis of detectability and statistical properties with respect to the reconstructed conducting region," Physiological Measurement, vol. 27, no. 5, pp. 249-259, 2006.

[26] P. C. Hansen, Rank-deficient and discrete ill-posed problems. Philadelphia: SIAM, 1998.

[27] Y. S. Shim and Z. H. Cho, "SVD pseduo-inversion image reconstruction," IEEE Transactions on Accoustics, Speech and Signal Processing, vol. ASSP-29, no. 4, pp. 904-909, 1981.

[28] N. G. Gençer, M. Kuzuoğlu, and Y. Z. İder, "Electrical impedance tomography using induced currents," Medical Imaging, IEEE Transactions on, vol. 13, no. 2, pp. 338-350, 1994.

[29] R. Wenxin, R. Guardo, and A. Adler, "Experimental evaluation of two iterative reconstruction methods for induced current electrical impedance tomography," Medical Imaging, IEEE Transactions on, vol. 15, pp. 180187, 1996.

[30] B. M. Eyüboğlu, A. Köksal, and M. Demirbilek, "Distinguishability analysis of an induced current EIT system using discrete coils," Physics in Medicine and Biology, vol. 45, no. 7, pp. 1997-2009, 2000.

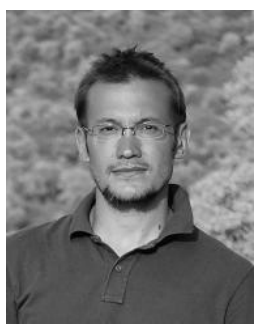

Doğa Gürsoy was born in Ankara, Turkey, in October 1981. He received the B.Sc. and M.Sc. degrees in electrical and electronics engineering from the Middle East Technical University (METU), Ankara, in 2004 and 2007, respectively and Ph.D. degree in electrical engineering from the Graz University of Technology (TUGRAZ), Graz, Austria, in 2010.

From 2006 to 2007, he was a Research Assistant in the Department of Electrical and Electronics Engineering, METU. From 2007 to 2010, he has been a Research and Teaching Assistant at the Institute of Medical Engineering, TUGRAZ. Since 2010, he has been working as a postdoctoral researcher at the TUGRAZ. His current research interests are medical imaging, computational physics and inverse problems.

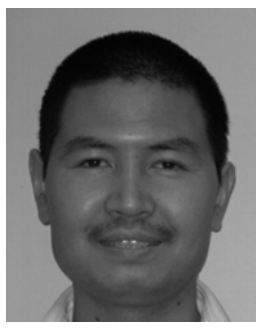

Yasin Mamatjan received the B.Sc. from Nanjing University, China in 1999, the M.Sc. from both Chalmers University of Technology in 2003 and Jnkping University, Sweden in 2005. He obtained his $\mathrm{Ph} . \mathrm{D}$. in Medical Electronics from University of Glamorgan, UK in 2009.

$\mathrm{He}$ was a researcher at Saab in Sweden and a Marie Curie Research Fellow/senior research scientist at Philips research laboratory in Germany. He had made research visits at core research labs in Italy and France. Currently, he is a postdoctoral fellow at the Carleton University in biomedical engineering, where he is working on improving electrical impedance tomography (EIT) algorithms.

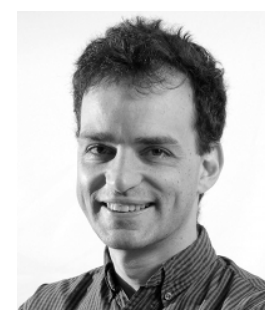

Andy Adler is professor and Canada Research Chair in biomedical engineering in Systems and Computer Engineering at Carleton University in Ottawa, Canada. His research interests are in: biometrics imaging and security systems, and the associated algorithms, measurement devices, and privacy and security aspects; and development of non-invasive biomedical measurement technologies and sensors, including the medical image and signal processing algorithms.

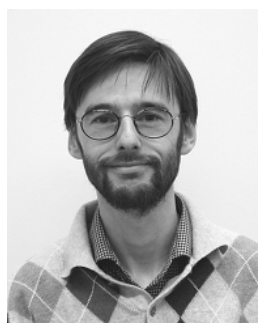

Hermann Scharfetter was born in Leoben, Austria, in June 1966. He received the degree of M.Sc. and Ph.D. in electrical engineering 1990 and 1995, respectively, both from the Graz University of Technology, Graz, Austria.

He was habilitated for Biomedical Engineering in 2000 and is currently Associate Professor at the Institute for Medical Engineering at the Graz University of Technology. His current research interests are preclinical and medical imaging methods based on near-field approaches like magnetic induction tomography and fluorescence diffuse optical tomography. 